\title{
EVALUATING THE POTENTIAL ROLE OF VACUOLIZATION IN ENHANCING OR INTERFERING WITH FISH VIRUS DETECTION AND REPLICATION
}

\author{
Phuc H Pham, Amreen Babujee, Fotini Papazotos, Niels C Bols ${ }^{\S}$ \\ Department of Biology, University of Waterloo, Waterloo, ON, N2L 3Gl, Canada
}

\begin{abstract}
Vacuolization is an often observed characteristic of virus infection on cell lines during cell based virus detection and isolation protocols and is commonly considered as a cytopathic effect (CPE) resulting from virus infection. However, caution should be exercised when vacuolization is used to indicate CPE as in addition to being induced by viruses, vacuolization in cells can occur spontaneously, as a response to cellular damage, during natural sequestration of materials and fluids, and fusion of many smaller vesicles formed by cellular processes such as autophagy and material transport. In vitro, additional vacuolization inducing factors during virus isolation may include use of antibiotic and antifungal, tissue preserving agents (RNA Later), temperature, and medium levels. Therefore, the goal of this research is to examine the capacity of these factors to induce cellular vacuolization during fish virus isolation procedures. Four cell lines used were EPC, CHSE-214 and two Atlantic salmon cell lines Asimf20 (Atlantic Salmon Intestinal Myofibroblast) and ASP309 (Atlantic Salmon Pituitary). Amphotericin B at concentrations between 2.5 to $10 \mu \mathrm{g} / \mathrm{mL}$ induced vacuoles in all cell lines except EPC. RNA Later at dilutions of 0.01 to 0.001 (v/w) induced vacuoles in all cell lines. Vacuoles were more likely to appear in cell lines when incubated at lower temperature $\left(4^{\circ} \mathrm{C}\right)$ and lower infection volumes. Additionally, the effects of vacuolization inducing agents on fish virus stability and replication was examined with two fish viruses, viral hemorrhagic septicemia virus (VHSV) and Chum salmon reovirus (CSV). RNA Later reduced the titre of enveloped VHSV by one $\log$ while having no observable effect on non-enveloped CSV. Due to the multifactorial induction of vacuoles in cells, care must be taken during virus isolation procedures to control or reduce alternative factors that may be causing vacuoles formation in cells to prevent misinterpretation of results.
\end{abstract}

\section{KEYWORDS}

Vacuolization, fish viruses, Atlantic salmon, ammonium, RNA Later

${ }^{\S}$ Corresponding author. Tel.: +1 5198851211 x33993; Fax: +1 5197460614.

E-mail address: ncbols@uwaterloo.ca (N.C. Bols) 